\title{
Concepciones y modelos subyacentes en la práctica pedagógica de los docentes cuyo desempeño es el mejor evaluado por estudiantes universitarios
}

\author{
Libia Villamizar-González ${ }^{\text {a* }}$ \\ aDocente Investigador, Universidad Simón Bolívar, Cúcuta. \\ Forma de citar: González, L., (2017). Concepciones y modelos subyacentes en la práctica pedagógica de los \\ docentes cuyo desempeño es el mejor evaluado por estudiantes universitarios. Perspectivas, 2(1). 28-42
}

Recibido: junio 12 de 2016

Aceptado: octubre 05 de 2016

\section{Palabras clave \\ Desempeño docente, práctica pedagógica, modelos pedagógicos}

Resumen: La forma en la que los docentes ejercen sus prácticas pedagógicas repercute con fuerza sobre el proceso de enseñanza aprendizaje. Es por eso que los docentes con mejor desempeño contribuyen en la creación de condiciones óptimas para el aprendizaje a partir de un entorno crítico natural, en el que se vale de sus destrezas para dar un mejor uso a la información que ellos quieren enseñar mediante estrategias que los estudiantes encontrarán atrayentes. Por otra parte, los estudiantes suelen distinguir entre sus profesores los que ejercen su labor con mayor empeño y efectividad. En este sentido, el objetivo central de la investigación consistió en caracterizar las prácticas pedagógicas de los docentes mejor evaluados por sus alumnos en la UFPS. El estudio se abordó desde el paradigma cualitativo, para lo cual se utilizó un diseño descriptivo de caso. Los sujetos de investigación fueron cinco docentes, uno de cada facultad y cuarenta y tres estudiantes que tomaron asignaturas con estos docentes. En la recolección de información se utilizaron dos técnicas: relatos de vida y entrevistas semiestructuradas, se indagó sobre las percepciones que tienen los docentes y estudiantes sobre las prácticas pedagógicas. Los hallazgos de la investigación reflejan que las prácticas de los docentes mejor evaluados se caracterizan por ser disciplinadas, planificadas y comprometidas, identificándose que los docentes no se apegan a nivel teórico y pedagógico a un modelo pedagógico determinado. También se encontró que los atributos reconocidos por los estudiantes como propios de un buen docente son la puntualidad, la responsabilidad, el compromiso y la actualización disciplinar y

* Autor para correspondencia

libiavillamizar27@hotmail.com 
pedagógica. En conclusión, las altas evaluaciones de los docentes por parte de sus alumnos están soportadas en la flexibilidad y la relación docente estudiante.

\section{Keywords}

Teaching performance, teaching methods, pedagogical models

\section{Palavras chave}

Desempenho docente, prática pedagógica, modelos pedagógicos

\section{Underlying conceptions and models in the pedagogical practice of} teachers whose performance is the best evaluated by university students

Abstract :The way teachers utilize their teaching methods has a strong impact on the teaching-learning process. Therefore, teachers who perform better in the classroom create the optimal conditions for learning from a critical natural environment, in which the teachers may apply their skills to employ teaching skills that utilize the information they want to teach, so that students may find their lessons more appealing. Nevertheless, students tend to easily identify which of their professors teach with greater effort and effectiveness. Accordingly, the central objective of this study was to characterize the teaching practices of the UFPS professor's which scored highest on the students' evaluations. The investigation was consistent with the qualitative paradigm, for which a descriptive case study design was used. The research subjects were five teachers (one from each faculty) and forty-three of their students. There were two techniques used to collect data: semi-structured interviews and hearsay, both concerning the perceptions that teachers and students have about teaching methods. The findings of the research demonstrate that the methods of the teachers with the more positive student evaluations are characterized by discipline, planning and commitment. The study also identified that all the teachers, on both a theoretical and educational level, did not stick to any particular pedagogical model. It also found that the attributes, as recognized by the students, of a good teacher, are punctuality, responsibility, being committed, disciplined and having a determined teaching approach. In conclusion, the students' high ratings of their teachers are founded in both the adjustibility and the student-teacher relationship.

\section{Concepções e modelos subjacentes na prática pedagógica de professores cujo desempenho é o melhor avaliado por estudantes universitários}

Resumo: A forma como os professores exercem suas práticas pedagógicas tem um forte impacto no processo ensino-aprendizagem. É por isso que os professores com melhor desempenho contribuem para a criação de condições ideais para aprender a partir de um ambiente crítico natural, que usa suas habilidades para fazer melhor uso da informação que eles querem ensinar 
através de estratégias que os estudantes encontrarão atraente. Por outro lado, os alunos muitas vezes distinguem entre seus professores aqueles que exercitam seu trabalho com maior esforço e eficácia. Nesse sentido, o objetivo central da pesquisa consistiu em caracterizar as práticas pedagógicas dos professores melhor avaliados pelos alunos na UFPS. O estudo foi abordado a partir do paradigma qualitativo, para o qual foi utilizado um desenho de caso descritivo. Os sujeitos da pesquisa foram cinco professores, um de cada faculdade e quarenta e três alunos que cursaram assuntos com esses professores. Na coleta de informações, foram utilizadas duas técnicas: histórias de vida e entrevistas semiestruturadas, e as percepções que os professores e alunos têm sobre práticas pedagógicas foram investigadas. Os resultados da pesquisa mostram que as práticas dos melhores professores avaliados são caracterizadas por serem disciplinadas, planejadas e comprometidas, identificando que os professores não aderem a um modelo pedagógico específico a nível teórico e pedagógico. Verificou-se também que os atributos reconhecidos pelos alunos como pertencentes a um bom professor são a pontualidade, responsabilidade, compromisso e atualização disciplinar e pedagógica. Em conclusão, as avaliações elevadas dos professores por seus alunos são apoiadas na flexibilidade e na relação professor-aluno.

\section{Introducción}

Caracterizar las prácticas pedagógicas de los docentes mejor valorados por sus estudiantes puede resultar de gran interés y relevancia para la pedagogía y las ciencias de la educación en general, pues distinguir y reconocer las características de la actuación profesional de los docentes que obtienen los más altos puntajes cuando son evaluados por sus permitiría reconocer lo que causa el éxito del docente, qué cualidades o particularidades distinguen el ejercicio pedagógico de estos docentes, qué hace el docente en el aula para que su desempeño sea relevante o no, cómo resuelve problemas y cómo enfrenta las situaciones que surgen en la labor docente, qué factores convergen para propiciar el éxito del docente, qué atributos o competencias particulares poseen.

Para conocer qué causa el éxito del docente entre sus alumnos se tuvieron en cuenta durante esta investigación una serie de categorías preliminares: a. Las prácticas pedagógicas, incluyendo su conceptualización, la relación docente estudiante, las estrategias de enseñanza aprendizaje, los contenidos de enseñanza y los métodos de evaluación. b. El modelo pedagógico que subyace en las prácticas pedagógicas. c. Las percepciones que los docentes mejor evaluados y sus estudiantes tienen de tales prácticas. d. Los atributos y competencias de los docentes exitosos.

Se consideró como eje central de la investigación las prácticas pedagógicas de los docentes, conocer de qué manera impactan en los procesos de enseñanza aprendizaje y cómo determinan el quehacer del maestro en el aula, haciendo relevante o no su desempeño Gamboa, A. A. (2016). Esta actuación se enmarca en un contexto más general en el que se entiende que la enseñanza "como toda practica social, contingente a unas circunstancias históricas y especiales determinadas, se encuentra penetrada por 
opciones de valor y, por tanto, hay que identificar su calidad en los valores intrínsecos que se desarrollan en la misma actividad" (Pérez, 1999:103). En relación con lo anterior, la calidad se evidencia mediante los recursos, contenidos y estrategias que configuran las prácticas pedagógicas de los docentes. Por ello es de gran importancia conocer lo que realmente realiza el maestro en el aula de clase, cómo afronta y resuelve problemas, qué estrategias y técnicas son las que le permiten mayores acercamientos con el estudiante, cómo utiliza el conocimiento científico y su capacidad intelectual, cómo establece relaciones con los diferentes saberes, y qué recursos didácticos utiliza, cómo crea o innova y mantiene la motivación del estudiante, es decir qué hace que la práctica pedagógica del docente sea relevante, percibida como exitosa, de alta calidad o desempeño excelente, para los actores principales del proceso: los estudiantes (Pérez, 1999).

La evaluación sobre los docentes brinda información de interés sobre cómo perciben los estudiantes a sus profesores en sus prácticas pedagógicas en el aula Gamboa, A. A. (2016). Para esta investigación se partió de la suposición de que estas prácticas deben ser significativas e impactar positivamente sobre el estudiante, y que se pueden considerar como prácticas pedagógicas significativas las que pueden ser objeto de investigación, reflexión y aprendizaje. Todo lo referenciado hasta el momento, sustentó la pregunta desencadenante de la investigación ¿cómo son las prácticas pedagógicas de los docentes mejor evaluados en la universidad Francisco de Paula Santander? Dar respuesta a este interrogante permitiría descubrir y describir las prácticas pedagógicas de los docentes mejor evaluados, reconocer lo que realmente los estudiantes valoran de sus docentes y lo que los lleva evaluarlos con los más altos puntajes. En este sentido se buscó descubrir las características particulares del quehacer docente, para reconocer en que está fundamentado el ejercicio pedagógico, reconocer los modelos, discursos y las formas particulares de establecer diálogos de saberes con sus estudiantes y cómo conquista el interés de sus discípulos, qué estrategias utiliza y le son eficaces frente en el proceso de enseñanza aprendizaje.

De otra parte, se ha procurado contar con una investigación que ofrezca una aproximación o que indique qué cualidades perciben los estudiantes o que atributos valoran de sus docentes, para interpretar y comprender las dinámicas que se desarrollan en las aulas de clase como producto de las prácticas pedagógicas del docente, que "siempre son un acto de creación, una actividad artística apoyada en el conocimiento y las experiencias pasadas" (Gimeno, 1999: 101). También se ha intentado posibilitar la identificación de qué aspectos en el quehacer pedagógico se consolidan como claves, con el fin de contribuir a que otros docentes aumenten la calidad educativa en la formación integral de los futuros profesionales. Sobre este particular se pronuncia Bain (2007: 58), que afirma que los mejores profesores de universidad crean lo que podríamos llamar un entorno para el aprendizaje crítico natural, en el que incluyen las destrezas y la información que ellos quieren enseñar mediante trabajos (preguntas y tareas) que los estudiantes encontrarán fascinantes, auténticas tareas que les provocarán curiosidad, que les motivarán a repensar sus supuestos y a examinar sus modelos mentales de la realidad.

La reflexión sobre cómo conseguir maestros y profesores motivados en mejorar o transformar las prácticas pedagógicas, que los lleve a pensar o crear nuevas maneras de intervenir en el quehacer educativo, o visibilizar alternativas para el avance pedagógico es de vital importancia en la educación , pues la labor del docente involucra o presume un "aprendizaje y actualización permanente, máxime cuando los vertiginosos y significativos cambios en la realidad sociocultural, en los contenidos de los programas, en las actitudes de los educandos, en los avances científicos y técnicos, constituyen un desafío a la función educadora".(Tébar, 2003: 191). Es decir la investigación también podría apoyar el desarrollo pedagógico y el fortalecimiento de la calidad educativa institucional sirviendo de punto de partida en la reflexión de los docentes y en el diseño de prácticas pedagógicas renovadas, modificadas, 
significativas y transcendentes en la labor educativa, que conduzcan a la formación de profesionales éticos, críticos, con gran sentido de la responsabilidad social. Desde esta mirada pedagógica, Suárez (2007) afirma que el docente como agente de la pedagogía, como un intelectual capaz de teorizar, dirigido por sus vivencias y el sentimiento profesional e intelectual que ejerce sobre el proceso educativo - formativo que orienta, identifica en qué procesos pedagógicos y dispositivos didácticos están soportadas sus prácticas pedagógicas y su compromiso por el desarrollo, crecimiento intelectual y personal del estudiante.

En coherencia con la exposición precedente y las preguntas de investigación, en esta investigación se planteó como objetivo caracterizar las prácticas pedagógicas de los docentes mejor evaluados en la Universidad Francisco de Paula Santander, a partir de las percepciones que sobre sus prácticas pedagógicas tienen los docentes y los estudiantes. En este sentido se pretende identificar los modelos pedagógicos que subyacen en las prácticas pedagógicas de los docentes. Asimismo, se busca describir las estrategias de enseñanza aprendizaje que hacen exitosas las prácticas pedagógicas de los docentes mejor evaluados.

\section{Materiales y métodos}

Para la satisfacción del propósito de la investigación se eligió el paradigma cualitativo. El diseño del estudio, concretamente, fue de naturaleza descriptiva interpretativa, pues un "estudio de caso descriptivo en educación [...] nos da luces de las realidades que viven nuestros docentes y alumnos en una situación particular" (Parra de Chópite, 1995: 10), es decir, el estudio permite apreciar la realidad para caracterizar las prácticas pedagógicas de los docentes mejor evaluados. Asimismo, desde la perspectiva naturalista, se describen las percepciones de los docentes y los estudiantes, que son las interpretaciones y significaciones que cada uno tiene de la realidad que viven en ese contexto. Es a lo que se refiere Merrian (citado por Parra de Chópite, 1995: 14) cuando afirma que "el mundo no es una cosa objetiva externa, sino una función de interacción y percepción personal

Las categorías conceptuales de las que se partió luego de la fijación de propósitos y revisión de la bibliografía fueron las siguientes: a) aspectos relevantes o elementos inherentes de las prácticas pedagógicas que permitieran de manera fiel y exacta la descripción y caracterización de las prácticas pedagógicas de los docentes mejor evaluados de la UFPS; b) que ofrecieran una descripción cabal del ejercicio pedagógico de los docentes desde las percepciones de los profesores y de los estudiantes; c) que develaran la realidad que sucede en el aula, es decir que se identificara el modelo pedagógico en el que los docentes soportan sus prácticas pedagógicas; yd) que se descubrieran las atributos y competencias que causan el éxito del docente mejor evaluado.

\subsection{Contexto e informantes clave}

El escenario donde se llevó a cabo la investigación fue la Universidad Francisco de Paula Santander, Sede Cúcuta, Institución de Educación Superior (IES) pública del orden departamental.

Los sujetos de investigación fueron los cinco docentes mejor evaluados por los estudiantes durante dos semestres consecutivamente, adscritos a las facultades de Ingeniería, Ciencias Empresariales, Ciencias Básicas, Ciencias Agrarias y del Ambiente y Ciencias de la Salud.

Para el estudio de caso, la selección de la muestra de sujetos de investigación es intencional y no existen reglas para el tamaño de la muestra (Parra de Chópite, 1995: 36). Los criterios de selección de los sujetos de investigación fueron: a) Docentes que obtuvieron resultados en la evaluación docente superior a 4.8 de forma consecutiva y continua por b) tres semestres académicos. Estudiantes que estuvieran cursando la asignatura con los docentes evaluados. La tabla 1 relaciona la cantidad de docentes y estudiantes que participaron en el estudio. 
Tabla 1

Docentes y estudiantes participantes

\begin{tabular}{ccccc}
\hline C & Informantes & Código & Docente & Facultad \\
\hline 10 & estudiantes & E:IM-1-10 & DH- 01 & Ingeniería \\
10 & estudiantes & E:CA-1-10 & DM-02 & Ciencias Empresariales \\
7 & estudiantes & E:CB-1-7 & DM-03 Básicas \\
6 & estudiantes & E:CAG-1-6 & DM-04 & Ciencias Agrarias \\
10 & estudiantes & E:CS-1-10 & DM-05 & Ciencias de la Salud \\
\hline 43 & Estudiantes & & 5 Facultades \\
\hline
\end{tabular}

Fuente: Autor

Los estudiantes participantes en la investigación fueron 43 en total: 10 estudiantes de Ingeniería Mecánica de diferentes semestres, 10 de Administración de Empresas, 7 de Ciencias Básicas, 6 de la Facultad de Ciencias Agrarias y 10 estudiantes de la Facultad de Ciencias de la salud.

\subsection{Instrumentos de recolección de la información}

En investigación cualitativa y en el estudio de casos, el investigador puede utilizar varias técnicas. Tal y como señalan Osses, Sánchez e Ibáñez (2006: 1), “en el proceso de recolección de datos, el investigador va acumulando numerosos textos provenientes de diferentes técnicas". Partiendo de esta premisa se optó por dos técnicas: los relatos de vida y la entrevista semiestructurada "en función de la saturación del discurso y del grado de profundidad que se quiera dar al estudio" (Blasco y Otero, 2008: $1)$.

Relatos de vida: Los relatos de vida son "un subgénero de la historia de vida, menos amplio y completo. Se trazan los rasgos más destacados, atendiendo a los aspectos que más interés tienen para el investigador." (Arjona y Checa, 1998: 3 ).

Entrevista semiestructurada: La entrevista semiestructurada (no estructurada o no formalizada) "es aquella en que existe un margen más o menos grande de libertad para formular las preguntas y las respuestas" (Sabino, 1992). En este tipo de entrevista, según afirman Jiménez y Rico (1998: 14 ) “el sujeto nos acerca a su mundo, a sus relaciones con el grupo primario (familia, escuela...), al contexto social y cultural que le rodea" (p.14). En el estudio de casos se permiten contenidos de "citas de entrevista y observaciones que ilustran la complejidad de una situación”.(Parra de Chópite, 1995: 8 ).

\subsection{Análisis de la información}

El análisis de los datos en la investigación cualitativa se realiza en el transcurso de todo el estudio. Luego de la definición teórica de las categorías del estudio, se diseñó la matriz reconstructiva con los relatos de vida de los docentes, de donde emergieron categorías, que brindaron importante información para la descripción de los resultados finales. Con la información obtenida de los docentes en sus entrevistas se elaboró una segunda matriz reconstructiva teniendo en cuenta las categorías teóricas de las percepciones. Las entrevistas grupales realizadas a los estudiantes se analizaron en las mismas categorías.

El análisis de la información transcrita y organizada en las matrices enunciadas, se realizó a través de la triangulación, que según Denzin (1970) es la combinación de dos o más teorías, de fuentes de datos y métodos de investigación en el estudio de un fenómeno particular. Cuando la triangulación se hace con datos se confrontan diferentes fuentes de datos en la investigación, y se realiza cuando concuerdan o discrepan estas fuentes (Pereyra, 
2007). En este sentido se confrontaron los relatos de vida y las entrevistas semiestructuradas de los docentes con las percepciones expresadas por los estudiantes en las entrevistas grupales y la literatura de los referentes teóricos del estudio. Al triangular de este modo la información se obtiene lo que Cisterna (2005) llama un corpus coherente, que muestra de forma organizada lo que será los resultados de la investigación.

\section{Resultados y Discusión}

A continuación, se hace una descripción de los principales hallazgos obtenidos del análisis de los relatos de vida de los docentes y de las entrevistas realizadas a los docentes y los estudiantes. Siguiendo el orden del análisis se describen cada una de las categorías obtenidas.

\subsection{Percepción de las prácticas pedagógicas por docentes y estudiantes}

Las percepciones que los docentes tienen de sus prácticas pedagógicas permiten caracterizarlas e identificar los modelos pedagógicos que subyacen en las mismas. De esta manera se aborda la primera categoría, exponiendo textos o párrafos expresados por los propios docentes en las entrevistas, hasta llegar a las percepciones que los estudiantes tienen de las prácticas pedagógicas de los docentes mejor evaluados. Los docentes expresaron:

Soy estricto, yo sé que algunos procuran evadirme, no matricular la materia conmigo. Yo ni les quito ni les doy, he sido lo más honrado posible. Yo los animo y le digo que ellos son muy capaces. DH-01.

Por su parte, los estudiantes perciben al Docente H-01 como un profesor experto, exigente y disciplinado:

Tiene un alto dominio de los temas, tiene mucha experiencia, una amplia trayectoria profesional, es muy exigente y disciplinado, propone que los estudiantes deben apropiarse de los conceptos básicos para que dominen los temas avanzados. (E: I M-2).

Desde la práctica pedagógica los estudiantes perciben a los docentes como profesionales comprometidos y de gran experticia:

Da ejemplo de que cuando uno quiere puede, el ejemplo de lo que debemos ser los administradores, ya que en su forma de expresarse, actuar y llevar a cabo las actividades (...) deja ver que es comprometida y con mucha experiencia. (E:CA-3).

Bain (2007 ) afirma que el docente es percibido como exitoso cuando logra ayudar al estudiante en su aprendizaje e influye positivamente en sus formas de pensar, sentir y actuar, con lo cual consigue resultados educativos muy buenos. Dicho de otro modo, logran movilizar los recursos de los estudiantes para que progresen en el proceso de aprendizaje, orientándolos para que se esfuercen, desarrollen comprensión superior y se impongan o asuman retos cognitivos, que les potencien en sus capacidades.

También se encontró que para los estudiantes es relevante la confianza que poseen en los conocimientos del docente, la seguridad que este transmite, la calidez y el trato humano que perciben en la práctica, la claridad de sus explicaciones y la argumentación de su saber, a la vez que la autoridad que perciben, acompañada de un trato agradable y el acompañamiento del proceso de aprendizaje. Esto se evidencia en expresiones como:

Es una docente humana, con calidad y calidez en su profesión, porque en la asignatura expresa conocimientos, calidad en el abordaje, y enseña de manera integral al estudiante, de manera que comprenda el entorno biopsicosocial. (E: CS$1)$. 
En este mismo sentido, los estudiantes valoran las clases bien explicadas y actualizadas. En sus palabras, ofrecen

Enseñanza actualizada, excelente manejo del tema a tratar, temas claros y bien explicados, se entienden todas las clases, da asesorías. (E:CS3).

También hacen referencia a los valores personales que perciben de la docente:

Es una docente con conocimientos excelentes sobre la profesión y como persona es muy amable, respetuosa, y nos ofrece un trato muy cálido, nunca se niega a sacarnos de una duda. (E:CS-2).

Según Wertheimer (citado por Carterette y Friedman, 1982) las percepciones son un estado subjetivo de donde se abstrae el mundo externo o hechos relevantes. Los docentes se perciben en sus prácticas pedagógicas abstrayendo sus propias experiencias, sus cotidianidades, aspectos que desde lo subjetivo consideran lo más relevante de su ejercicio pedagógico, es decir, que lo asumen como un "proceso de extracción y selección de información relevante" (Oviedo, 2004). En lo que concierne concretamente a las practicas pedagógicas, Achilli et al (citados por Castro, 2006) enfatizan que estas se caracterizan por la relación del docente, alumno y conocimiento, escenario en el que se comunican y se conjugan expectativas, sentimientos y valoraciones.

Como resumen parcial, los sujetos de la investigación se perciben como docentes estrictos, puntuales, exigentes, disciplinados, organizados, serviciales, constantes, conocen las asignaturas que orientaran, organizan y desarrollan sus prácticas pedagógicas planificando las clases, buscan la actualización de los temas, guían y orientan a los estudiantes, les hacen observaciones en procura de su aprovechamiento y mejoramiento del proceso de aprendizaje, ofrecen apoyo constante, son persuasivos, escuchan y dialogan con los estudiantes, e incluso asumen otros roles frente a las necesidades que estos presentan en el aula.

\subsection{Modelo pedagógico subyacente en las prácticas pedagógicas de los docentes mejor evaluados}

En relación con la identificar los modelos pedagógicos que subyacen entre los sujetos del presente estudio, no se precisa un modelo en que basan o sustentan sus prácticas. Quizá no tengan claro el modelo como tal, pero la forma como orienten las clases y la intención que tienen al desarrollarlas se relata de diferentes formas.

Es relevante identificar que a través de la práctica pedagógica el docente propicia espacios para las preguntas, aclaración de dudas, participación y reflexión, facilitando el aprendizaje de los estudiantes. A este respecto se expresan los estudiantes:

Uno ve la importancia de que el estudiante aprenda a comprender, que no sea simplemente un receptor, una máquina, porque muchas veces uno ve que un resultado no lo piensan, no importa si metieron a calculadora y les dio un valor estúpido sin ningún análisis. Eso no es lo que uno busca. DH-01.

A ese respecto dice otro estudiante:

Con él se puede profundizar porque da la confianza para preguntar y resolver dudas, lo hace con agrado, le gusta que razonemos cuando estamos en la clase, que analicemos. (E: IM-7).

Flórez (1999) señala que un modelo pedagógico es la representación de las relaciones en el arte de enseñar, las formas de ejercitar una clase. Para Abarca (2007), tiene que ver con la forma como se hacen las cosas. Desde la perspectiva cognitivo constructivista, "el estudiante construye y reconstruye sus conocimientos a partir de la acción. Este conocimiento se enlaza a las estructuras mentales 
de los estudiantes" (Moreno, 2004: 56). Esto quiere decir que el eje del modelo se encuentra en el aprender haciendo, y que el docente es un facilitador que contribuye para desarrollar las potencialidades de los estudiantes. La intención consiste en desarrollar las habilidades del pensamiento para que el estudiante puede progresar en su estructura cognitiva:

Ahora, por la forma como oriento la clase, que por lo general lo hago por medio de un conversatorio, yo me demoro en un párrafo hasta veinte minutos, porque yo doy el título, y pregunto qué comprenden. Yo doy la definición y luego empiezo a profundizar. (DM-02).

Los estudiantes coinciden con la docente en la forma en que ejercita su labor:

Como la profesora da las clases, se puede participar, y meterse uno en el cuento. Es muy organizada, como presenta la temática. Las clases hacen que se piense en el tema. (E:CA5).

Schon (1983) habla de la diferencia en la ejecución de labores donde se tiene "conocimiento en la acción" y "reflexión en la acción”. Así, desde la mirada de los expertos, la pedagogía en ejercicio, o sea, en la clase, se basa en la reflexión, que "es un proceso en el que se integran actitudes y capacidades en los métodos de investigación, de modo que el conocimiento de la realidad surge de la experiencia" (Dewey, citado por Pérez, 1999: 413). Así lo expresan tanto la docente como los estudiantes.

Los docentes manifestaron que ejercitan su práctica pedagógica de diferentes formas:

Yo creo que soy reconductista. Si veo que funcionan los estímulos, les doy premios, les llevo hasta dulces, libros, si son buenos, o si realizan prácticas excelentes. Pero lo más importante es donde ellos aplican el conocimiento, la parte conceptual se supera en la aplicabilidad. (M-03).
Con referencia en lo expresado por la docente, un estudiante suyo afirma:

Considero que tiene cualidades y métodos de enseñanza prácticos en su pedagogía. (E: C B-1).

Con las docentes M-04 y M-05 se encontró que sus formas de orientar las asignaturas está basada en la planificación y organización de los temas que ofrecen magistralmente o por medio de guías y talleres:

Me gustan las cosas prácticas. Para ellos es mejor y que lo apliquen a la vida profesional. (DM-04).

Refiere un estudiante en el mismo sentido:

Desde el principio es muy clara en decir cómo se orientará la asignatura, pide que preguntemos $\mathrm{y}$ aclaremos las dudas o las posibles inquietudes que tengamos. (E: CAG-1).

En una dirección semejante se expresa otra de las docentes:

Mi práctica pedagógica es bastante planeada, desde finalizado el semestre se mira cuáles han sido los logros, las dificultades, las particularidades que tienen los estudiantes. (DM-05).

Desde la percepción de los estudiantes de las Ciencias de la Salud existe coincidencia con el juicio de la docente M-05:

Es una profesora que se presta al diálogo y pone las reglas claras antes de iniciar la asignatura. Tiene una manera muy buena de hacer entender las teorías y motivar a los estudiantes a la investigación y la lectura. (E:CS-E). 
Los docentes no reconocen, concretan ni describen los modelos pedagógicos que subyacen en las prácticas de los docentes, pero al hablar del modo en el que orientan sus clases los develan. El docente H-01 se ajusta al modelo cognitivo constructivista, pero teniendo en cuenta que se centra más en el desarrollo del pensamiento y las potencialidades de los estudiantes, entonces fundamenta más su pedagogía en el desarrollo cognitivo, principios integrados por el pensamiento de varios autores como Vigotsky, Piaget, Bruner. La docente M-02 con su práctica planificada, organizada y basada en su experiencia personal se orienta a la práctica reflexiva, que le permite pensar en la acción que realiza y mejorarla. En el modelo conductista se logra ubicar la docente M-03 pues sus relatos precisan los estímulos y refuerzos que aplica a sus estudiantes para el logro del aprendizaje, y finalmente las docentes M-04 y $\mathrm{M}-05$, desde las diferentes actividades que realizan con los estudiantes para el progreso y desarrollo del aprendizaje, se ajustan con la fundamentado del modelo constructivista.

\subsection{Relación docente estudiante}

Se encontró que los docentes entrevistados visibilizan a sus estudiantes de diferentes maneras, desde los estudiantes inmaduros y algunas veces inconscientes en los primeros semestres, a estudiantes inquietos, con grandes expectativas y sueños, estudiantes muy habilidosos y altamente intelectuales, algunos con falta de entrenamiento y desarrollo de sus habilidades, con falencias en el aprendizaje por ausencia de estímulos en el ambiente psicosocial en que se desarrollaron o como consecuencia de la educación que recibieron en el colegio en el que se formaron. Reportan asimismo estudiantes dinámicos, trabajadores, con gran sentido crítico y estudiantes 'bellos', como los describe una de los docentes, personas humanas con fortalezas y debilidades. Todos los docentes coinciden en que sus relaciones con los estudiantes se dan en términos de receptividad, confianza, armonía, respeto, exigencia y disciplina, pero también de amistad, apoyo en las dificultades y muchas veces en situaciones privadas ofrecen orientación personal.

Los estudiantes señalan como dificultades:

La exigencia en la asignatura, la disciplina y lo estricto en calificar y el cumplimiento de los horarios. (E: I M-9).

Pero también se encontró estudiantes que expresaron:

No se relaciona de la misma forma con todos los estudiantes, no hay confianza y no se muestra amigable, por lo tanto no hay motivación, uno se siente prevenido para acercarse. (E: CB-3).

Se encontró que los estudiantes valoran la forma como los tratan los docentes. Sobre este aspecto dice un estudiante:

Es una persona respetuosa, suave en su forma de ser, no arma problema por nada, no como otros que son una cagada y siempre se la quieren montar al estudiante. (E: CA-6).

En cuanto a si la relación docente estudiante se basa en el ejercicio de alguna forma de poder, el común denominador entre los docentes fue la manifestación de un no rotundo, pues consideran que el trato que otorgan a sus estudiantes es de respeto y de reconocimiento de personas iguales.

Para Mélich (2001), la relación entre docente y estudiante ocurre entre personas. Aunque desempeñen roles de distinta índole, son humanas. Al docente corresponde iniciar y mantener la dinámica que se genere en el aula, procurando que sea de simpatía, confianza, y aceptación. Desde el proceso de enseñanza aprendizaje es fundamental que se efectúe "la tarea de optimizar el crecimiento de los alumnos" (Vélez, 2006). En el reconocimiento de valores que fortalecen la relación docente estudiante, "el respeto, el reconocimiento y la aceptación de la diferencia" es esencial (Martínez, 2000, citado por 
Vélez, 2006: 9). Tanto profesores y estudiantes valoran las relaciones de respeto y cercanía que las partes logran tejer, situación que es favorable para el proceso enseñanza aprendizaje y crecimiento personal de los estudiantes, ya que el docente se compromete desde su modo de pensar y "de vivir y por ello su forma de facilitar el crecimiento de los alumnos está marcada por lo que es valioso para él. Educar implica ponernos en relación con el otro, lo cual supone dejar una huella en el otro" (Vélez, 2006: 9)

\subsection{Estrategias de enseñanza}

En las prácticas pedagógicas esta categoría tiene relevancia por cuanto configura particularmente la didáctica utilizada por el docente, es decir su habilidad, creatividad e innovación en las técnicas particulares que emplea en su proceso de enseñanza o labor pedagógica para captar el interés o motivar al estudiante, es decir la habilidad metodológica para el desarrollo de las clases, en función de presentar de manera atractiva, adecuada y acertada los conocimientos, que las estrategias empleadas tengan la fundamentación científica del saber disciplinar.

Al indagar sobre las estrategias de enseñanza que con mayor frecuencia utilizan los docentes entrevistados expresaron:

Utilizo mucho las películas, videos, me encanta mucho el crucigrama, los previos en crucigrama, los saco de la rutina. Inicialmente todo el mundo se enoja, pero ya luego entienden, porque los enseño a pensar. (DM-02).

Para Corredor, Pérez y Arbeláez (2009), tanto los docentes como las universidades enfrentan retos cuando introducen métodos innovadores en las estrategias de enseñanza aprendizaje, pues tales innovaciones no solo deben centrarse en el aspecto del aprendizaje sino también en la formación de las personas. En referencia a las metodologías utilizadas para las clases, docentes y estudiantes coinciden en que los métodos como los software, internet y ayudas visuales funcionan dentro de los objetivos del logro del aprendizaje. Algunos estudiantes desean que se pudiera innovar en cuanto a otros métodos que el docente diseñara como potencialmente efectivos. En este orden de ideas, Carpio de los Pinos (2007: 11) señala que "de poco valen los métodos didácticos, por innovadores que sean, sino producen una implicación efectiva de los estudiantes". Dicho de otro modo, los métodos deberán enganchar o captar el interés de los estudiantes para que el aprendizaje se dé de manera efectiva.

\subsection{Contenidos de enseñanza}

Introducir los contenidos de enseñanza es tarea principal del docente. Un proyecto educativo solo tiene sentido si en las practicas pedagógicas se delimitan con claridad cuáles son los contenidos que se pretenden desarrollar para la formación del futuro profesional. Dicho de otro modo, los contenidos llenan de sentido y significado dichas prácticas. A través de la presentación de contenidos, los docentes enfatizan no solo en los conocimientos pertinentes del saber teórico y del saber hacer para la formación profesional, sino que se comprometen con el saber ser o la formación para la vida, es decir, orientan sus asignaturas para la construcción de mejores seres humanos, de personas sensibles a las necesidades de los contextos en los que mueven, en la educación personal de sí mismos.

A este respecto, los docentes señalan que de los contenidos es lo que más interesa al estudiante:

La aplicación es lo que ellos buscan aprender, y cuando llegan a la aplicación ven que es mucho más difícil que la sola conceptualización o la mecanización de la solución de los mismos problemas. (Docente M-03).

Para los estudiantes es de gran relevancia que los contenidos y las prácticas guarden relación con su etapa profesional futura. Desde este punto se encontró que efectivamente la intención que los docentes dan a los contenidos, sí colma las 
expectativas de la mayoría de los estudiantes. Para Tanner y Tanner (1980, citado por Gimeno, 1999: 142 ) los temas del currículum van de la instrucción a la acción, es decir, se desarrollan a través de la práctica. Esto quiere decir que los contenidos cobran vida y relevancia en la contextualización y en la practicidad.

\subsection{Métodos de evaluación}

Para los docentes sujetos de la investigación, la evaluación no es un elemento nuclear de preocupación, pues supone una actividad más entre todas las que constituyen su ejercicio docente. En este sentido, asumen la evaluación como el proceso que se deriva del compromiso que tiene el estudiante para lograr su avance y progreso académico. Los docentes toman la evaluación como uno de los requisitos que deben cumplir por norma institucional y consideran su utilidad en cuanto les permite reconocer las habilidades que se han potenciado en el proceso de aprendizaje. En la información recabada durante la investigación se encontró que los docentes realizan la evaluación como se describe en el estatuto del estudiante, y los instrumentos de evaluación que más utilizan son los previos escritos, orales, talleres, trabajos escritos, exposiciones, ensayos, quizzes, trabajos en grupo y de manera práctica. Al indagar como evalúan a los estudiantes, algunos señalaron que lo hacen de manera descriptiva cualitativa.

Los estudiantes refirieron también los diversos métodos de evaluación descritos por los docentes. Además, mencionan que las evaluaciones realizadas se ajustan a los temas que han sido abordados académicamente y con los criterios que con anterioridad han sido expuestos por el docente. Las evaluaciones fueron descritas por los estudiantes como desafiantes cognitivamente. En sus propias palabras:

Los previos con él son arrechos. Uno tiene que tirarles como se dice cacumen, porque es muy estricto al calificar. (E: I M-6).
También tienen sus propias opiniones acerca de cómo evalúan sus profesores y los sentimientos que despiertan cuando se hacen conscientes de la probabilidad de fracaso:

Con el profesor...... se tiene que estudiar. De lo contrario uno puede pasar todo del previo aullando. Son difíciles y califica duro. (E: I M-2).

En consonancia con estos testimonios, Jackson (1975) señala que las evaluaciones simbolizan y resaltan los procesos post-activos de la enseñanza. En otros términos, lo que sobreviene de lo que ha pasado en un periodo de enseñanza. Tal y como lo expresan los docentes y los estudiantes, es algo normal que de deriva de la práctica pedagógica y del proceso de aprendizaje, y tanto docentes como estudiantes están conscientes de su ineludible realización.

\subsection{Atributos y competencias de los docentes extraordinarios o exitosos}

Los docentes que obtienen altos puntajes en la evaluación que realizan los estudiantes se proyectan comoreferentesdeéxito paralacomunidadacadémica. Los atributos, cualidades, calidades y valores que son percibidos por los estudiantes hacen que les otorguen los más altos puntajes en la valoración de las prácticas, promoviendo positivamente su imagen y valoración. Desde esta perspectiva se encontró que los docentes describen que los atributos o cualidades no radican en un aspecto en particular sino en la unión de varios aspectos, factores o cualidades que según ellos, quizá es lo que valoran sus estudiantes.

Desde los atributos o competencias particulares se encontró que los docentes hicieron referencia a cualidades o valores específicos que los caracterizan y que piensan que son percibidos por los estudiantes como de gran valor para su ejercicio pedagógico y que les permiten acercarse a sus estudiantes sin mayor dificultad, es decir, cualidades que se convierten en los mejores aliados para lograr mejores ambientes para el aprendizaje y favorecer la relación docente 
estudiante, y por tanto desarrollar el proceso de enseñanza aprendizaje en condiciones de aceptación, responsabilidad y compromiso, donde los docentes son referentes de estos valores y los estudiantes responden adecuadamente a estos por el respeto $\mathrm{y}$ otros valores que el docente les inspira.

En este orden de ideas los docentes entrevistados refirieron cuestiones como la siguiente:

Trato de tener buenas relaciones con los estudiantes. La parte humana la manejo mucho. Me gusta enseñar, tengo vocación. Generalmente uno no está porque cayó ahí como docente, sino porque me gusta y es lo he hecho siempre.(DM-04).

Entre las singularidades reportadas por los docentes se encuentran la planeación, organización, confianza, solidaridad, flexibilidad, compromiso, carisma, responsabilidad, constancia, disciplina, experticia, respeto, puntualidad, trato amable y considerado, reconocimiento del otro como persona, orientador o consejero y amigo de sus estudiantes. Como se ve, el factor humano se constituye como la principal característica de conexión entre todas las actividades realizadas con los estudiantes.

Se encontró que los estudiantes valoran cualidades específicas que no perciben en otros docentes, tales como la forma particular de tratarlos, de enseñar, la autoridad, disciplina, experiencia, aspectos que los estudiantes reconocen como características relevantes En este sentido expresaron:

Realmente es un profesor que lo da todo, se esmera porque uno entienda, da la confianza necesaria para preguntar, uno se da cuenta que tiene mucha experiencia, se actualiza, además es calidad de persona. (E: IM-5).

Yo creo que su personalidad agradable, su actualización constante, sus clases son muy motivadoras y ella es muy carismática, hacen que uno valore otras cosas. Aunque nos exija una estricta puntualidad, su seguridad, eso la hace una docente exitosa. (E: CS-9).

Bain (2007: 35) afirma que los docentes exitosos "conocen muy bien sus disciplinas y son académicos, artistas o científicos en activo". Además, los profesores exitosos conocen muy bien la historia de sus disciplinas, es decir su desarrollo epistemológico. Los docentes sujetos del presente estudio muestran en sus prácticas gran coherencia con la opinión del autor, pues según expresan ellos mismos y también sus estudiantes, tienen un profundo conocimiento de sus disciplinas y constantemente se actualizan para mayor comprensión de sus disciplinas, favoreciendo sus prácticas pedagógicas, además de enseñar o apoyar el desarrollo académico de sus estudiantes, haciendo las asignaturas interesantes y motivadoras. Los docentes extraordinarios 0 exitosos, dice Bain (2007), saben lo que desean o quieren intelectualmente de sus estudiantes. En este sentido lo que los docentes mejor evaluados de la UFPS anhelan de sus estudiantes es un conocimiento profundo de sus disciplinas, y lo ayudan a que lo consigan durante su proceso de aprendizaje.

Zabalza (2003), por su parte, considera que de las competencias comunicativas de los docentes deben permitir transmitir mensajes con afecto. Más todavía, según el autor, la característica principal del docente es transmitir 'pasión'. En este sentido los estudiantes participantes de la investigación relataron que algunos de sus docentes se expresaban en sus asignaturas con pasión, tratando de motivar, inspirar e implicar a sus alumnos en el conocimiento de las mismas. Segura (2005), por su parte, afirma que un buen profesional ha de poseer cualidades mínimas como persona, más aún cuando se es docente. En este sentido, tanto profesores como estudiantes expresaron las cualidades que los distinguen tales como disciplina, puntualidad, responsabilidad, flexibilidad, compromiso, constancia, planificación, organización, creatividad, solidaridad, empatía, exigencia, don de gentes, amabilidad y gran calidad humana. En resumen, los profesores poseen las 
cualidades que se espera en un docente, tanto como profesional cuanto como persona.

\section{Conclusiones}

Los docentes mejor evaluados de la universidad UFPS tienen prácticas heterogéneas que, sin embargo, se caracterizan, por ser planificadas, no improvisadas. Su disciplina de trabajo y compromiso hacen que el docente se programe, revise y actualice los conocimientos, lo cual les permite tener un conocimiento profundo de sus disciplinas. Otra característicarelevante tiene que ver con las relaciones interpersonales que el docente logra tejer en el aula con sus estudiantes. El carisma, la buena disposición y el acercamiento cálido del docente al estudiante permiten lograr el aprecio, respeto y confianza de los alumnos. Los docentes perciben que sus prácticas son valoradas como exitosas por los estudiantes debido a factores tales como la flexibilidad, la puntualidad, la calidad humana, el trato amable y respetuoso, el reconocimiento del otro, sus actitudes solidarias y su experticia en el ejercicio profesional y docente. De todos estos elementos, otorgan relevancia al factor humano como principal atributo de su relación con los docentes.

En relación con los modelos pedagógicos que subyacen en las prácticas de los docentes mejor evaluados, se identificó que el quehacer de los profesores en el aula está relacionado con varios de ellos, y que ninguno de los docentes se enfoca de forma estricta en un modelo en particular. Sus prácticas, en efecto, reúnen atributos diversos. En los datos recabados, los docentes no describieron de manera precisa en qué modelos sustentan sus prácticas.

Las prácticas pedagógicas de los docentes mejor evaluados de la UFPS también se caracterizan porque utilizan gran variedad de estrategias de enseñanza aprendizaje. Más todavía: perciben que la pluralidad de métodos forman parte de su éxito docente. Entre estas técnicas se reconocieron las explicaciones sencillas o profundas, el desarrollo de guías, la elaboración de talleres, búsqueda de solución de problemas, los estudios de caso, aclaración de dudas, la indagación, documentación. Asimismo, se valen de las herramientas tecnológicas tales como los software, el uso de internet, los recursos audiovisuales (películas, videos, y diapositivas) e introducen a sus estudiantes en complejidades que les sirvan de desafío cognitivo.

Los estudiantes identifican a los docentes mejor evaluados con cualidades específicas que no perciben en otros. Entre ellas se encuentran un alto dominio de los temas, amplia experiencia, ser referentes de éxito, exigentes pero muy humanos y flexibles, presentan los planes de trabajo que con antelación han planificado, organizado y actualizado, son docentes que llevan los conocimientos de la teoría a la práctica, ofrecen ayuda al estudiante en sus dificultades y lo apoyan en su proceso académico, utilizan métodos de enseñanza apropiados, realizan explicaciones convincentes y aclaran las dudas que tienen los estudiantes y fomentan la investigación y la práctica. También son percibidos como docentes comprometidos, con gran sentido de la disciplina y puntualidad. Pero lo que para los estudiantes resulta más significativo es el carácter humano e sus profesores, que les permiten establecer con ellos relaciones de amistad y profundo aprecio.

\section{Referencias}

Arjona, G. y Checa, O. (1998). Las historias de vida como método de acercamiento a la realidad social. Gazeta de Antropología, 14. Disponible en http://digibug.ugr.es/handle/10481/7548

Bain, K. (2007). Lo que hacen los mejores profesores universitarios. Valencia: Publicacions Universitat de València.

Blasco, H y Otero, G. (2008) Técnicas conversacionales para la recogida de datos en investigación cualitativa: La entrevista (II).Nure Investigación, 34. Disponible en http://www. fuden.es/FICHEROS_ADMINISTRADOR/F_ METODOLOGICA/formet_34.pdf

Carpio de los Pinos, C. (2008) Métodos de enseñanza aprendizaje aplicables en el magisterio en el 
marco del Espacio Europeo de Educación Superior. Docencia e Investigación, XXXII: 22. Disponible en https://ruidera.uclm.es/xmlui/ handle/10578/8137

Carterette, E., \& Friedman, M. (1982). Manual de percepción :raices históricas y filosóficas. México : Trillas.

Cisterna, F. (2005). Categorización y Triangulación como Procesos de Validación del Conocimiento en Investigación Cualitativa. Theoria, 14(1), 6171.

Contreras, J. (2014). Manual para la Elaboración e Implementación de un Modelo de Evaluación por Competencias. Estados Unidos de América: Palibrio LLC.

Corredor, M.; Pérez, A. y Arbeláez, L. (2009). Estrategias de enseñanza de aprendizaje. Colombia: UIS.

Denzin, N. K. (1970). Sociological Methods: A Source Book. Chicago: Aldine Publishing Company.

Flórez, R. (1999). Evaluación, pedagogía y cognición. Santafé de Bogotá D.C.: McGrawHill Interamericana. S. A.

Gamboa, A. A. (2016). Docencia, investigación y gestión: Reflexiones sobre su papel en la calidad de la educación superior. Perspectivas. 1(1). 8190.

Gimeno, S. y Pérez, G. (1999). Comprender y transformar la enseñanza. Madrid: Morata.

Jiménez, Ma. Á y Rico, A.M. (1998). Los relatos de vida como métodos de investigación Intercultural. Disoonible en sedll.org/es/admin/uploads/.../ Jimenez_Jimenez, Angeles_y_otra.pdf'

Mélich, J-C. (2001). La ausencia del testimonio: ética y pedagogía en los relatos del Holocausto. Barcelona: Anthropos.

Moreno, H. (2004). Pedagogía y otros conceptos afines. Bogotá: SEM.

Parra de Chópite, B. (1995). Estudio de caso cualitativo en la investigación educativa. Mérida: Universidad de los Andes.

Pereyra, L (2007). Integración de metodologías cuantitativas y cualitativas: Técnicas de triangulación. Disponible en ief.eco.unc.edu. ar/files/workshops/2007/09oct07_lilipereyra work.pdf|

Osses,S.;Sánchez,I.\&Ibáñez,F.(2013).Investigación cualitativa en educación: hacia la generación de teoría a través del proceso analítico. Estudios pedagógicos, 32 (1): 119-133. Disponible en https://scielo.conicyt.cl/scielo.php?script=sci arttext\&pid=S0718-07052006000100007

Oviedo, G. (2004). La definición del concepto de percepción en psicología con base en la teoría. Revista de Estudios Sociales, 18: 89-96. Disponible en https://revistas.uniandes.edu.co/ doi/abs/10.7440/res18.2004.08

Sabino, C. (1992) El proceso de investigación. Caracas: Panapo .

Schon, D. (2011). Una Páactica Profesional Reflexiva en la Universidad. Compás Empresarial, 3(5), 14-21.

Segura, M. (2005). Competencias Personales del Docente. Revista ciencias de la Educación, 2(26), 171-190.

Stake, R. (1998). Investigación con estudio de caso. Madrid: Morata.

Suárez, M. (2007). El saber pedagógico de los profesores de la Universidad de Los Andes Táchira y sus implicaciones en la enseñanza. Tesis doctoral inédita. Barcelona: Universitat Rovira i Virgili. Disponible en http://www.tdx. cat/handle/10803/8922

Tébar, B. (2003). El perfil del profesor mediador. Madrid: Santillana.

Taylor, S.J. y Bogdan, R. (1992). Introducción a los métodos cualitativos en investigación. La búsqueda de los significados. Barcelona: Paidós

Vélez, V. O. (2006) La relación profesor alumno en el aula como espacio de la formación ética en la Universidad. Revista Digital de Investigación Universitaria, 2(1):21. Disponible en http://revistas. upc.edu.pe/index.php/docencia/article/view/25/528

Zabalza, M. (2003). Competencias docentes del profesorado universitario. Calidad y desarrollo profesional. Madrid: Narcea. 